\title{
Diffraction-unlimited three-dimensional optical nanoscopy with opposing lenses
}

\author{
Stefan W. Hell ${ }^{\star}$, Roman Schmidt and Alexander Egner
}

\begin{abstract}
The resolution of far-field optical microscopy stagnated for a century, but a quest began in the 1990s leading to nanoscale imaging of transparent fluorescent objects in three dimensions. Important elements in this pursuit were the synthesis of the aperture of two opposing lenses and the modulation or switching of the fluorescence of adjacent markers. The first element provided nearly isotropic three-dimensional resolution by improving the axial resolution by three-to sevenfold, and the second enabled the diffraction barrier to be overcome. Here, we review recent progress in the synergistic combination of these two elements which non-invasively provide an isotropic diffraction-unlimited three-dimensional resolution in transparent objects.
\end{abstract}

$\mathrm{D}$ espite the enormous resolution afforded by electron and scanning probe microscopy, far-field optical microscopy has maintained its key role in a number of areas, particularly in the life sciences. The reason is a series of key advantages of imaging with propagating fields of light, such as the non-invasive access to the interior of (living) cells, and the sensitive detection of macromolecules by fluorescence tagging. In fact, far-field fluorescence microscopy would be almost ideal for non-invasively investigating the three-dimensional (3D) interior of transparent objects in biology and material sciences if it could discern details that are substantially smaller than half the wavelength of light, $\lambda$. However, until not very long ago, realizing nanometre-scale resolution with conventional lenses and focused visible light seemed to be impossible because of diffraction ${ }^{1,2}$.

The limiting role of diffraction stems from the fact that the full-width half-maximum (FWHM) of the intensity point-spread function (PSF) $h(\mathbf{r})=h(\rho, z)-$ that is, the spatial light intensity pattern in the focal region of an objective lens - extends over at least $\Delta \rho \approx \lambda /(2 n \sin \alpha)>180 \mathrm{~nm}$ in the focal plane and $\Delta z \approx \lambda /$ $\left(n \sin ^{2} \alpha\right)>450 \mathrm{~nm}$ along the optic axis, with $\alpha$ denoting the semiaperture angle of the lens and $n$ denoting the refractive index of the medium. In the PSF, $\mathbf{r}$ is the position vector in the sample space, and $\rho, z$ are its coordinates in a cylindrical coordinate system. Features that are closer together than these dimensions can yield a (fluorescence) signal at the same time, so the individual signals cannot be discerned. Likewise, imaging a point object from the focal region of the lens to a camera is governed by a similar PSF, $h_{\mathrm{em}}(\mathbf{r})$, which blurs the spatial coordinate of the object. Therefore, until the advent of the first concrete concepts for breaking the diffraction resolution barrier in the early 1990s, it had been widely accepted that optical imaging at the nanoscale would not be viable with freely propagating light waves. The only pathway seemed to be offered by near-field optical microscopy, confining the lightspecimen interaction with a nanosized tip ${ }^{3-5}$ (see also the review article on page 388 of this issue ${ }^{6}$. However, tip-confined nonpropagating light waves may not image the interior of transparent objects and hence they are largely limited to imaging surfaces. Because this technique also relies on collecting and amplifying nonpropagating waves, similar practical limitations currently also apply for imaging with a metamaterial lens of negative refractive index ${ }^{7}$. Meanwhile, the early concepts of diffraction-unlimited farfield optical microscopy ${ }^{8-10}$ have been validated ${ }^{11,12}$, expanded ${ }^{13-15}$ and applied ${ }^{16}$. Moreover, they have been recently complemented with other powerful techniques ${ }^{17-20}$ that have greatly extended the range of implementation and application of diffraction-unlimited far-field optical nanoscopy.

\section{Aperture increase and signal switching}

Several advancements have contributed to attaining the current record values of far-field optical 3D resolution of $<50 \mathrm{~nm}$. Yet when analysing their basic physical principles, two unrelated elements come into sight, which, harnessed in various optical arrangements, have provided major leaps in 3D far-field optical resolution: increasing the solid aperture angle of the microscope by the coherent use of two opposing lenses ${ }^{10,21-26}$; and the breaking of the diffraction barrier by switching or modulating the signalling (fluorescence) ability of markers that are closer than the diffraction limit to enable their separate registration ${ }^{8,9,13,17-19,27}$.

The microscope's total aperture is increased by counter aligning two large angle lenses and coherently adding the spherical wavefront caps of the excitation light at the sample, or of the emitted fluorescence light at a common detector ${ }^{21,22}$. With lenses featuring a semiaperture angle $\alpha$ greater than about $65^{\circ}$, this approach virtually mimics the use of a nearly full solid angle of $4 \pi$ and concomitantly removes the asymmetry along the optic axis in focal spot formation, which is the actual reason for the poorer resolution along the optic axis $(\Delta z>2.5 \Delta \rho)$ in single-lens imaging. The coherent synthesis of the spherical wavefront caps of opposing lenses is the essence of scanning 4Pi microscopy, where either the excitation photons interfere at the sample (4Pi type A) and/or each emitted fluorescence photon interferes with itself at the detector (4Pi type B and C), after having simultaneously passed through both lenses ${ }^{22,28}$. As a result, the axial FWHM of $h(\mathbf{r})$ or $h_{\mathrm{em}}(\mathbf{r})$ is reduced by three- to fourfold over the typical axial FWHM obtainable with a single high-angle lens, and the main maximum is more spherical. Sharpening both $h(\mathbf{r})$ and $h_{\mathrm{em}}(\mathbf{r})$, as in 4Pi type C, improves the axial resolution up to about $70-100 \mathrm{~nm}$ (refs 22,28,29). The related concept, $\mathrm{I}^{5} \mathrm{M}$ (refs $23,25,30$ ), and its extension $I^{5} S$ (ref. 26), which adds laterally structured illumination, also coherently collect fluorescence photons through both lenses thus attaining a similar or even 1.5 -fold better $3 \mathrm{D}$ resolution in parallelized camera-based imaging. The significantly improved axial and hence nearly isotropic 3D resolution obtained through the spherical wavefront synthesis drastically improved the $3 \mathrm{D}$ imaging of cells, albeit within the limits set by diffraction.

By enabling sequential detection of adjacent 'identical' fluorescent features irrespective of their distance, the diffraction limit can be overcome fundamentally $y^{8,9,13,17-19,27,31}$. This can be done by modulating or switching the signalling ability of these features. The 
switching of a marker's ability to fluoresce, or to signal in general, implies a manifest transfer of the fluorophore from a signalling (fluorescent) 'on' state, A, to a non-signalling (non-fluorescent) 'off' state, B, or vice versa, such that only one of the states is noticeably occupied ${ }^{13,27}$. One way of realizing (fluorescence) switching is to prohibit one of the two states, say A. This is possible by subjecting the fluorophore to a light intensity that causes a saturated optical transition from A to B, depleting A. If the fluorophore happens to end up in A for some reason, it is so rapidly transferred to $\mathrm{B}$ that its dwell time in $\mathrm{A}$ is negligible. If the depletion of $\mathrm{A}$ is predominant and not contended by adverse optical transitions, the probability that the fluorophore is in the state A simply scales with $\exp \left(-I / I_{s}\right)$ (refs 12,27). I denotes the applied light intensity and $I_{s}$ is a fluorophore-characteristic (saturation) intensity that depends on the lifetime of the two states.

For example, exposing the fluorophore to an intensity of $I>I_{\mathrm{s}}$ induces stimulated emission from the fluorescent state to the ground state and effectively prohibits the fluorescent state. Hence the fluorophore is essentially confined to its ground state, which is equivalent to switching the fluorophore off ${ }^{8}$. It is instantly switched on again when the stimulating beam is interrupted. Conversely, a saturated excitation depleting the ground state switches fluorescence on to its maximum level, and back off again if the beam is interrupted $^{14,15}$. Mechanisms that demand less intense beams and lower $I_{s}$ include the switching of the fluorophore between (meta) stable fluorescence-active and fluorescence-inactive states ${ }^{13,31}$, such as the transfer between the fluorescent singlet and the metastable dark triplet state ${ }^{9}$ (flip of an electron spin), or between conformational (cis-trans) fluorescent and non-fluorescent states in organic fluorophores and photoactivatable fluorescent proteins ${ }^{13,17-19,31}$ (position flip of fluorophore atoms). Other options for switching the signalling ability of the fluorophore are changes in binding states, including that of diffusible fluorophores ${ }^{32}$.

As all these mechanisms have been used for overcoming the diffraction barrier, one can classify the various nanoscopy methods according to the switching or fluorescence modulation mechanism used $^{27}$. However, upon analysing the way in which the switching is implemented and the position of the fluorophores registered, they fall into two distinct categories: in the targeted mode, the coordinates at which the fluorophore is switched (to A or B) is defined at any point in time; whereas in the stochastic category, individual fluorophores are switched randomly in space ${ }^{27}$. The archetypes of the first category are stimulated emission depletion (STED) and ground state depletion (GSD) microscopy, whereas that of the latter is the concept called (fluorescence) photoactivation localization microscopy (PALM ${ }^{17,33}$ or FPALM ${ }^{19,34}$ ) or stochastic optical reconstruction microscopy (STORM) ${ }^{18,35}$ (see also the commentary article on page 365 of this issue $)^{36}$. The related concept PALMIRA ${ }^{20}$ switches single molecules stochastically both in space and in time.

\section{Resolving by targeted signal switching}

In the targeted (STED-like) mode, defining where the fluorophore is in $\mathrm{A}$ or $\mathrm{B}$ is accomplished by forcing the fluorophores to reside in one of the two states, say in B, by applying an intensity $I(\mathbf{r})$, and therefore prohibiting the other state $(\mathrm{A})$, say in proportion to $\exp \left[-I(r) / I_{s}\right]$. Importantly, $I(\mathbf{r})$ is structured such that it features at least a single deep minimum, ideally a zero, at one or more predefined coordinates, $\mathbf{p}_{\mathrm{i}}$, in space: $I\left(\mathbf{p}_{\mathrm{i}}\right)=0$. Because the resolution is to be increased in three dimensions, the zero is bordered by a doughnut-shaped intensity maximum $I_{\mathrm{m}}{ }^{\rho}:=\max [I(\rho, 0)]$ in the focal plane, and two peaks $I_{\mathrm{m}}{ }^{\mathrm{z}}:=\max [I(0, z)]$ along the optic axis. By adjusting $I_{\mathrm{m}}{ }^{p}, I_{\mathrm{m}}{ }^{2}$ $>I_{\mathrm{s}}$, the fluorophore can remain in A exclusively at $\mathbf{p}_{\mathrm{i}}$, and its immediate proximity in which $H(\mathbf{r}):=\exp \left[-I(\mathbf{r}) / I_{s}\right]$ is non-negligible. In this high-intensity approximation, $H(\mathbf{r})$ does not depend on $h(\mathbf{r})$ and assumes the role of the (fluorophore-state-dependent) effective
PSF of the imaging modality. Close to the zero, we can approximate $I(\mathbf{r})$ quadratically by writing $I(\mathbf{r}) \approx(2 \pi n / \lambda)^{2}\left[I_{\mathrm{m}}{ }^{\rho}\left(\beta^{\rho} \rho\right)^{2}+I_{\mathrm{m}}{ }^{z}\left(\beta^{z} z\right)^{2}\right]$ with geometric parameters $\beta^{\rho}$ and $\beta^{z}$ quantifying the steepness of the well ${ }^{37}$ in the radial and axial direction, respectively. Typically, $\beta^{\rho} \approx 0.5$ for a high-angle lens doughnut and $\beta^{z}=1$ for a flat standing wave. As a measure of the lateral and axial resolution, the FWHM of $H(\mathbf{r})$ is derived as

$$
\Delta \rho, \Delta z \approx \frac{\lambda}{\pi n \beta^{\rho, z}} \sqrt{\frac{\ln 2}{I_{m}^{\rho, z} / I_{s}}}
$$

From $I_{\mathrm{m}}{ }^{\rho}, I_{\mathrm{m}}{ }^{\mathrm{z}}>>I_{\mathrm{s}}$ it follows that $\Delta \rho, \Delta z<<\lambda /(2 n \sin \alpha)$. In this scheme, detectable features or molecules that are further apart than $\Delta \rho$ or $\Delta z$ are forced to differ by their state, which makes them separable for the time period in which this state gradient is maintained in space. Translating the zero through the sample sequentially brings them to the detectable state (say A), which allows an image of resolution $\Delta \rho$ or $\Delta z$ to be set up. In contrast, those fluorophores lying within $\Delta \rho$ or $\Delta z$ can emit their signal at the same time. Therefore, the total signal pertinent to a given coordinate pixel can be tuned through $\Delta \rho$ and $\Delta z$. More importantly, by varying $I_{\mathrm{m}}{ }^{\rho}$ and $I_{\mathrm{m}}{ }^{\mathrm{z}}$ between 0 and $\infty$, one can theoretically tune the resolution of $\Delta \rho$ and $\Delta z$ between the diffraction limit and the molecular scale.

Concretely, in STED microscopy ${ }^{8,11}$, the diffraction-limited focal spot formed by the beam of light exciting the fluorophores is overlapped with a 'holey' focal intensity distribution $I_{\text {STED }}(\mathbf{r})$ with $\lambda$ at the red edge of the emission spectrum of the fluorophore, so as to render stimulated emission as the predominant event in comparison to undesired excitation by $I_{\text {STED }}(\mathbf{r})$ or spontaneous emission. At coordinates where $I_{\text {STED }}(\mathbf{r})>>I_{\mathrm{s}}$, the fluorophore spends virtually no time in the excited state $(\mathrm{A})$ and hence effectively remains off (in $\mathrm{B}$ ), even if it is subject to the stream of photons provided by the excitation beam. The spatial region in which the fluorophore can be active, that is, effectively reside in $\mathrm{A}$, is given by equation (1). Note that because $I_{\mathrm{m}}{ }^{p}, I_{\mathrm{m}}{ }^{\mathrm{z}} \gg I_{\mathrm{s}}$ is assumed here, the unity in the square root of the denominator encountered in other versions of this equation $^{27,37}$ can safely be ignored.

\section{Separating objects by stochastic single molecule switching} In the stochastic (PALM-STORM-like) switching and read-out mode $\mathrm{e}^{17-20,33-35,38-41}$, individual fluorophores that are initially in a dark state B turn up in a bright state A at unknown coordinates $\mathbf{p}_{\mathrm{i}}$. The state $A$ is such that it is able to place $m>>1$ detected photons (for example, through repeated excitation $A \leftrightarrow A^{*}$ ) on a camera, mapping out $h_{\mathrm{em}}(\mathbf{r})$. This scheme does not require an $I(\mathbf{r})$ featuring a zero, but a state A that yields $m>>1$ detected photons. By considering the local fluorophore concentration in the adjustment of the excitation intensity so that only one molecule is in state A within range $\lambda /(2 n \sin \alpha)$, the unknown coordinate $\mathbf{p}_{\mathrm{i}}$ is then calculated ('localized') from the centroid of $h_{\mathrm{em}}(\mathbf{r})$ with subdiffraction precision $\Delta \rho \approx \lambda /(2 n \sin \alpha \sqrt{ } m)$ in the focal plane ${ }^{17-19}$. Finally, the fluorophore is switched off again from $A \rightarrow B$, so that an adjacent molecule can be read out using the same cycle $B \rightarrow A \rightarrow B$. If the molecule is read out only once, the initial and the final dark state $B$ need not be identical; the final state $B$ can be a 'bleached' state. The image is assembled mathematically by localizing a representative number of molecules. We note that switching between two states using a saturated optical transition is an optically nonlinear phenomenon. The molecular switching and onset of $m$ photons emitted and detected from the same molecule is also a highly nonlinear (of $m$ th order) phenomenon.

In any case, the two switching-based imaging modalities are complementary ${ }^{27}$. Imaging in the targeted mode can be very fast, because the molecular coordinate $\mathbf{p}_{\mathrm{i}}$ is known and a few photons are enough to indicate the presence of an object at the known coordinate. In addition, the average number of jointly detectable 


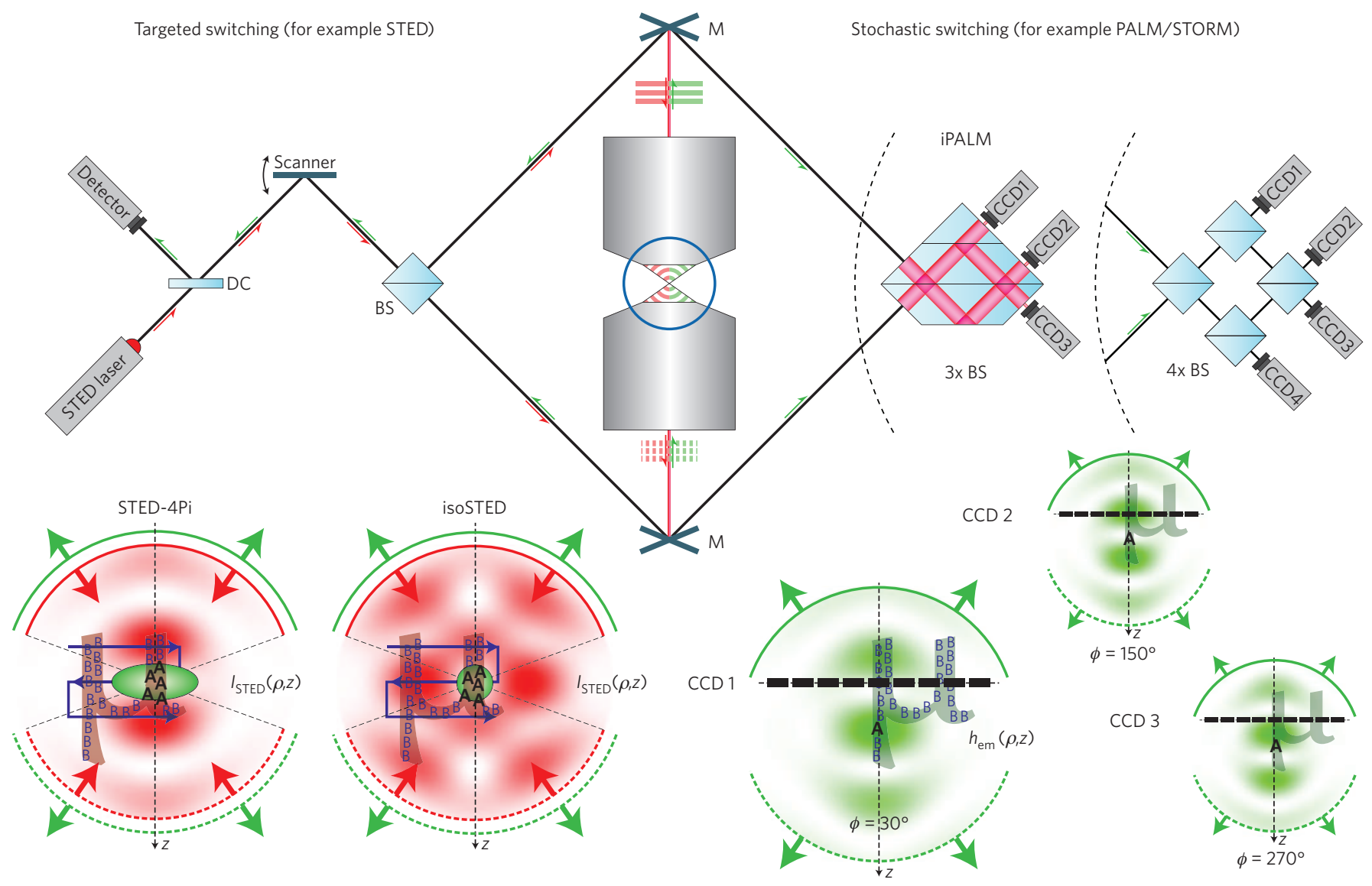

Figure 1 | Maximizing 3D resolution in far-field fluorescence nanoscopy by expanding the illumination and/or the detection aperture of the microscope with opposing lenses. The common focal region of the opposing lenses (blue circle) is sketced in the lower-left and lower-right panels for both imaging modalities. Left: targeted switching modality exemplified by STED microscopy implemented with a beam scanner. The STED laser beam is split into coherent parts so that they interfere destructively at the focal region rendering an $I_{\text {STED }}(r)$ with a central zero. The PSF describes the region in which the fluorophore is allowed to reside in the fluorescence 'on' state A, with FWHM $\Delta \rho$ and $\Delta z$ as given by equation (1). Fluorophores outside this (green) region are forced by $I_{\text {STED }}(\boldsymbol{r})$ to reside in the dark state $B$. The concept can be extended to other switching mechanisms between two states. Nanoscopy images are gained by scanning the (green) region through the transparent object (here a ' $\mu$ ') in 3D. Right: In the stochastic fluorophore switching modality, such as PALM or STORM, molecules are switched on and off individually, and the $m>>1$ photons emitted from the on-state A are used for 3D localization. Interferometric PALM (iPALM) or singlemolecule switching (SMS) combine the two spherical wavefront caps pertinent to fluorescence emission with a set of splitters (three in iPALM), re-dividing them such that partial wavefronts with distinct equidistant phase differences (for example, $\phi=30^{\circ}, 150^{\circ}, 270^{\circ}$ in iPALM) are created. An emitting fluorophore produces three different intensity patterns on the cameras, CCD1, CCD2, and CCD3, placed after each splitter outlet. These patterns represent magnified lateral $(x, y)$ cross-sections of a 4Pi detection PSF at the corresponding $\phi$, as indicated in the panels beneath the scheme. Owing to the strong dependence of their central and total brightness on the $z$ coordinate, these xy patterns localize the emitter (in state A) with improved sensitivity in $z$, but at the same time allow the localization in the xy plane. The triple-splitter used in PALM is an elegant implemention of the minimum number of splitters required for this detection scheme, which can also be implemented using four cascaded splitters and four camera detection areas (top right). Differences between the targeted and the stochastic modalities are that the first (for example, isoSTED) usually uses focused beams for excitation or fluorescence activation, whereas the latter (for example, iPALM) conveniently illuminates the sample in the wide field. DC, dichroic mirror; BS, beam splitter; $M$, mirror.

fluorophores can be adjusted by varying $\Delta \rho$ and $\Delta z$. A strength of the stochastic mode is that a spatially structured $I(\mathbf{r})$ is not required and each fluorophore has to undergo only a single cycle of $B \rightarrow A \rightarrow B$ to contribute to the image ${ }^{27}$. In contrast, the targeted mode requires optically induced multiple cycling, which is often challenged by molecular degradation. However, irrespective of the particular strengths and weaknesses of each modality, using just a single lens always implies that a large spectrum of the solid aperture angle is not covered. Therefore, coherently using opposing lenses automatically provides an improved axial and more isotropic 3D resolution in both the targeted ${ }^{10,12,42}$ and the stochastic switching approach $^{43,44}$ (Fig.1). For all these reasons, in the most recent efforts to maximize the 3D resolution of STED ${ }^{45}$ and $\mathrm{PALM}^{44}$, coherently using opposing lenses has (re-)assumed an important role.

\section{Targeted switching using opposing lenses}

In a targeted mode, such as STED microscopy, counter-propagating spherical wavefronts can concretely be used to create an $I(\mathbf{r})=I_{\text {STED }}(\mathbf{r})$ featuring a local minimum with an axial FWHM that is slightly larger than $\lambda /(4 n)$ (refs 10,12,42). The minimum has a larger intensity gradient, $\beta^{z}$, than can be created by destructive interference using a single wavefront cap, owing to the faster change of the relative phase in the interference of counterpropagating beams. For this reason, an early implementation of STED with opposing lenses, called STED$4 \mathrm{Pi}$ microscopy ${ }^{10,12}$, enabled $\Delta z=35-55 \mathrm{~nm}$, and hence made nanoscale imaging of bacteria cell membranes and microtubuli in fixed cells possible ${ }^{42}$. However, as $\beta^{p}<<\beta^{z}$, STED-4Pi microscopy did not really improve the resolution in the focal plane. Therefore, the most recent dual-lens implementation of STED, called isoSTED micros- 
a

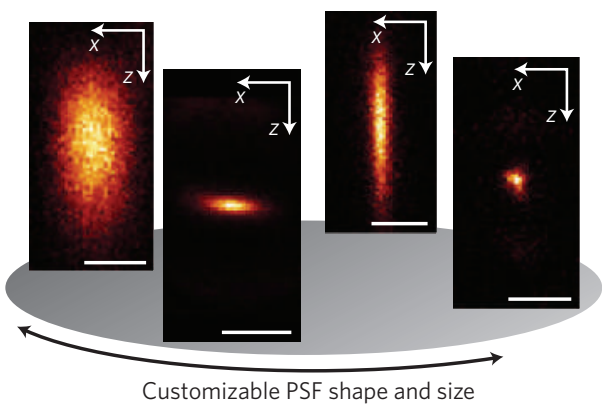

b
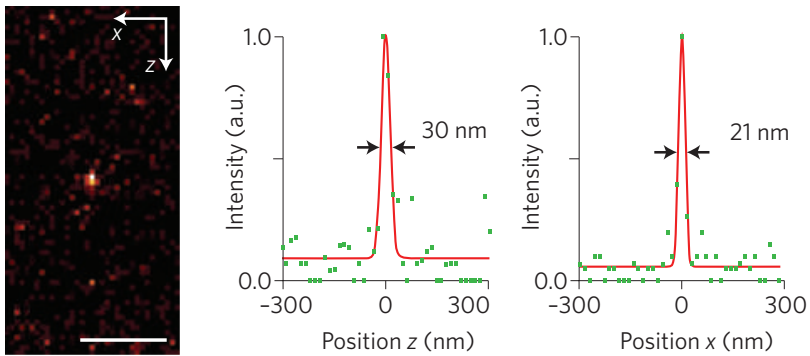

Figure 2 | PSF shaping in a targeted fluorophore switching modality.

a, By using the proper switching intensity distribution $I_{\text {STED }}(\mathbf{r})$, the PSF $H(\mathbf{r})$ of the imaging system - given by the subdiffraction region in which the fluorophore can reside in an emitting state - can be tailored to optimize signal and imaging speed. Panels compare PSFs of (from left to right) the standard confocal, an oblate STED-4Pi PSF, a prolate STED-PSF produced by a toroidal $I_{\text {STED }}$ with a doughnut-shaped cross-section in the focal plane, and a spherically shaped isoSTED-PSF. b, Currently, PSF dimensions of down to $\Delta z=30 \mathrm{~nm}$ axial and $\Delta \rho=21 \mathrm{~nm}$ lateral FWHM indicate that the effective PSF volume can be squeezed by at least three orders of magnitude with respect to its confocal counterpart. All PSFs were probed with fluorescent beads in the same setup: bead diameter $40 \mathrm{~nm}(\mathbf{a}),<20 \mathrm{~nm}$ (b) (Crimson fluorescent microspheres, Invitrogen, Carlsbad, California, USA), excitation at $635 \mathrm{~nm}$ and STED at $775 \mathrm{~nm}$ using synchronized $20 \mathrm{MHz}$ pulses, lens semiaperture angle $\alpha=74^{\circ}$, refractive index $n=1.52$. Scale bars: $250 \mathrm{~nm}$. Panels 1, 2 and 4 in a are reproduced with permission from ref. 48. (c) 2009 ACS. Part of $\mathbf{b}$ is reproduced with permission from ref. 49. (c) 2009 ACS.

copy $^{45}$, operates with focal intensity distributions $I_{\text {STED }}(\mathbf{r})$ featuring a spherical hole at the centre, thus enabling 'diffraction-unlimited' isotropic resolution in three dimensions, with $\Delta \rho=\Delta z \rightarrow 0$ (Fig. 1).

In $4 \mathrm{Pi}$ microscopy of type $\mathrm{B}$ and $\mathrm{C}^{22,28}$ and in $\mathrm{I}^{5} \mathrm{M}^{25,26}$, the coherent detection of the spherical wavefronts of photon emission results in an approximately fourfold faster change in phase difference in the photon self-interference at a common detector, when the emitter is changing its position along the optic axis. As a result, the axial FWHM of the main maximum of the detection PSF $h_{\mathrm{em}}(\mathbf{r})$ decreases by approximately fourfold. When localizing the axial position of individual emitters, the narrower main maxima produced with a $4 \mathrm{Pi}$ system improve the axial localization by the same factor ${ }^{46}$. However, when the fluorophores fall into the adjacent interference minima, they are not detected. One remedy is to scan the narrower 4Pi PSF along the $z$ axis while keeping the phase difference constant ${ }^{47}$. Another elegant solution is to measure the phase difference directly by phase-shift interferometry, as in the method called interferometric single-molecule switching ${ }^{43}$, or interferometric PALM (iPALM) ${ }^{44}$ (Fig. 1).

A unique benefit of a targeted mode such as STED is that it allows shaping of the PSF $H(\mathbf{r})$ through the parameters $\beta^{\rho} I_{\mathrm{m}}{ }^{\rho}$ and $\beta^{z} I_{\mathrm{m}}{ }^{\mathrm{z}}$, whereby almost arbitrary combinations of $\Delta \rho$ and $\Delta z$ can be attained. The overview in Fig. 2a compares the effective PSF $H(\mathbf{r})$ of a standard confocal fluorescence microscope with those PSFs that have so far been rendered by STED: the oblate PSF of a STED-4Pi
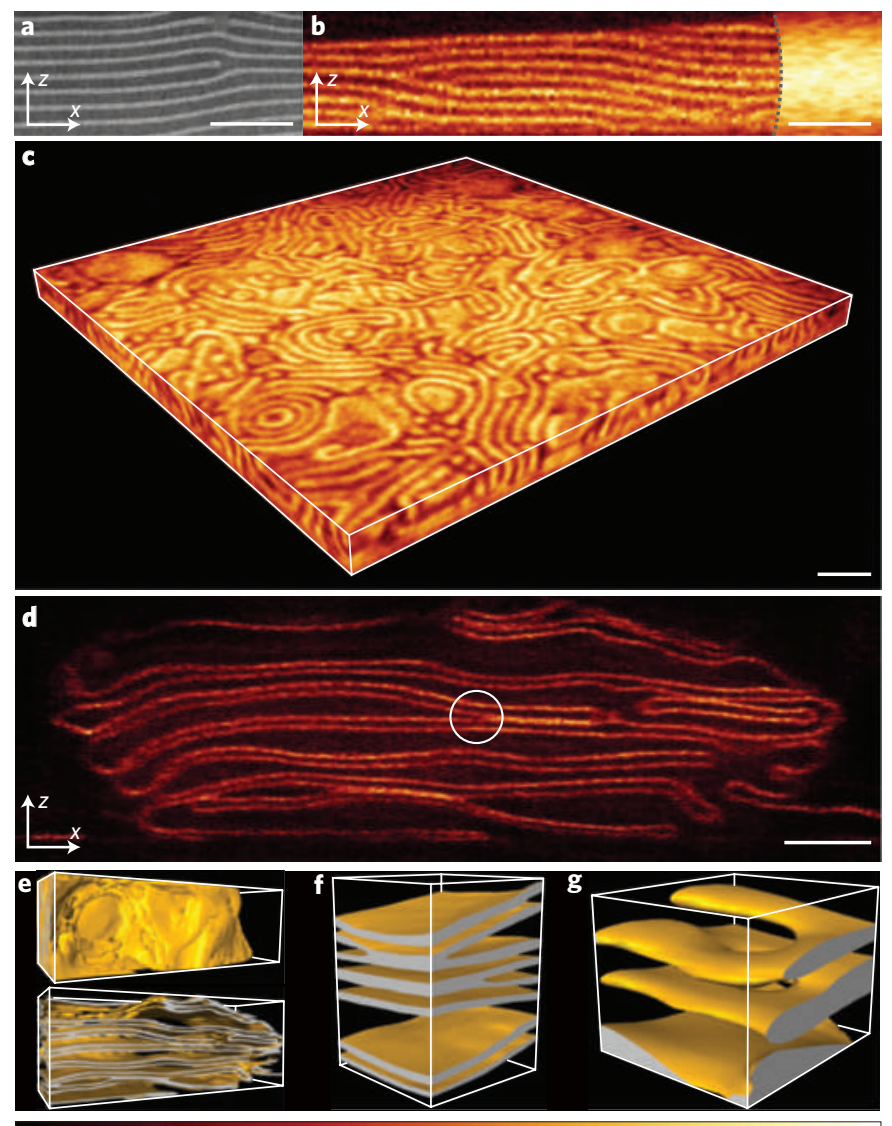

Intensity (a.u.)

Figure 3 | STED microscopy provides in situ access to 3D morphological information of nanostructured block copolymers. a, TEM image confirming the lamellar morphology of a polystyrene-block-poly(2vinylpyridine) polymer. $\mathbf{b}$, Whereas the confocal reference (right) provides no details, scanning with an oblate STED-4Pi-PSF (left) faithfully reveals the observed nanostructure. c, More isotropic morphologies, such as the bicontinuous structure shown, are revealed by means of a spherical isoSTED PSF. $\mathbf{d}$, Cross-sectional image of a mesoporous morphology induced by selectively swelling the vinyl-pyridine phase, subsequent to quaternization. The unzipped layers lining the pores (circled) are on average over half the size of the intact ones. $\mathbf{e}$, Perspective views of the corresponding binarized data stack. $\mathbf{f}$, Close-up of a selected area showing unzipping of the swollen domains, which line the pores. $\mathbf{g}$, Visualization of a helicoidal screw dislocation. Scale bars: $500 \mathrm{~nm}(\mathbf{a}-\mathbf{c})$ and $1 \mu \mathrm{m}$ (d). Images $\mathbf{a}, \mathbf{b}$ and $\mathbf{d - g}$ are reproduced with permission ref. 48. (c) 2009 ACS.

scheme $^{12}$ that also applies coherent dual-lens illumination to avoid axial sidelobes, the prolate PSF of a single-lens confocalized STED microscope using a cylindrical doughnut $I_{\text {STED }}(\mathbf{r})$ (ref. 16), and the isotropic PSF generated with a dual-lens isoSTED scheme ${ }^{45}$, recorded with the same setup and a fixed set of lenses $\left(\alpha=74^{\circ}\right)$, fluorophores and wavelengths. The PSF exemplified in Fig. $2 b$ displays an axial FWHM of $\Delta z=30 \mathrm{~nm}$ and $\Delta \rho=21 \mathrm{~nm}$ in the lateral direction. The reduction in PSF extent from the confocal reference to isoSTED by 10 -fold in the focal plane and 15 -fold along the $z$-axis highlights the recent progress in improving $3 \mathrm{D}$ resolution all-optically - that is, just by exploiting suitable optical transitions between molecular states. Although a volume larger than the confocal PSF is exposed to excitation photons, the dye is only able to reside in the excited state in the volume of the isoSTED PSF, represented by $H(\mathbf{r})$, which is 1,500 times smaller than that of the confocal PSF; in the remaining part, its ability to fluoresce is turned off by the STED beam. We 
note that the resolution in an image can be further augmented by deconvolution, which also benefits from the doubled number of detected photons.

This engineering of the PSF shape enables the adaptation of $\Delta \rho$ and $\Delta z$ and, hence, of the resolution and signal strength to the structure being imaged ${ }^{48}$. This is exemplified by fluorescence images (Fig. 3) of the self-assembled nanopattern formed by a di-block copolymer, in which one of the polymer phases is selectively labelled with an organic fluorophore. Imaging such nanostructures in three dimensions, for example the morphology shown in Fig. 3a, usually involves invasive forms of electron or scanning probe microscopy. In contrast, the oblate PSF of the refined STED-4Pi arrangement renders the lamellar nanostructure non-invasively (Fig. 3b). Block copolymer patterns without directional preferences (Fig. 3c) are best imaged with an isotropic PSF, here of $\Delta \rho, \Delta z<50 \mathrm{~nm}$. The technique facilitates the $3 \mathrm{D}$ in situ imaging of copolymer structures that would be difficult to image by established techniques. The massive gain in detail over confocal imaging proves that isoSTED and STED-4Pi nanoscopy are powerful non-invasive methodologies for $3 \mathrm{D}$ imaging of polymers at the nanoscale ${ }^{48}$.

The emerging importance of dual-lens fluorescence nanoscopy for the life sciences is exemplified in Fig. 4. All we know about the inner architecture of mitochondria, the powerhouses of the cell, is from electron microscopy, which in the 1970s revealed them as tubules of 200-400 nm diameter, formed by an inner and an outer membrane $^{2}$. By virtue of its isotropic $3 \mathrm{D}$ resolution of $<50 \mathrm{~nm}$, isoSTED microscopy has recently mapped out the distribution of the protein Tom 20 residing in the outer mitochondrial membrane ${ }^{45,49}$, clearly delineating its 'hollow' tubular shape (Fig. 4c). The Tom complexes represent the sites where cellular proteins enter the mitochondria. Until recently ${ }^{49}$, electron microscopy has also been required to image the highly convoluted inner membrane that harbours the $\mathrm{F}_{1} \mathrm{~F}_{0} \mathrm{ATPase}$ molecules pivotal to energy generation and folds into so-called cristae ${ }^{2}$. By scanning an $\sim 30$-nm-diameter isoSTED PSF directly through the equatorial plane of a mitochondrion in a whole fixed cell and recording immuno-labelled $\mathrm{F}_{1} \mathrm{~F}_{0} \mathrm{ATPase}$, the cristae were disclosed noninvasively (Fig. 4d,e) (ref. 49). The optical visualization of these central structural elements of mitochondria highlights the emerging ability of far-field fluorescence nanoscopy, and STED microscopy in particular, to image the substructure of organelles and eventually their 3D dynamics in living cells.

\section{Stochastic switching with opposing lens detection: iPALM} In interferometric single-molecule switching or iPALM ${ }^{44}$, axial super-resolution is gained by recombining the fluorescence wavefronts by a set of preferably three to four splitters re-dividing the two emission wavefronts, such that partial wavefronts with fixed but different equidistant phase differences, $\phi$, are created. The interference patterns rendered by individual emitters on a camera are mathematically represented by magnified lateral cross-sections of 4Pi-detection $\mathrm{PSF}^{22}$, which are calculated by adding the spherical fluorescence wave fields considering the specific $\phi$ (ref. 43). They exhibit maxima and minima that are narrower and steeper by approximately fourfold compared with the main maximum of a single lens, and improve the localization in the $z$ axis accordingly. The use of three to four detection PSFs with different but fixed $\phi$ elegantly avoids ambiguities and blind spots (produced by the minima at a given $\phi$ ) and reduces the calculation of the $z$ position to the calculation of the ratio of the signal of the three to four detection channels.

This scheme works well along a $z$-slice thickness of $\sim \lambda / 2 n$ on the $z$ axis. The cross-sections of the 4Pi PSF recorded on the cameras are summed to localize the emitter laterally or analysed for a refined 3D localization. The doubling of the number $m$ of detected photons augments the localization precision ideally by $\sqrt{ } 2$-fold in all directions $s^{43,44}$. By localizing the same single source with $m \approx 1,200$
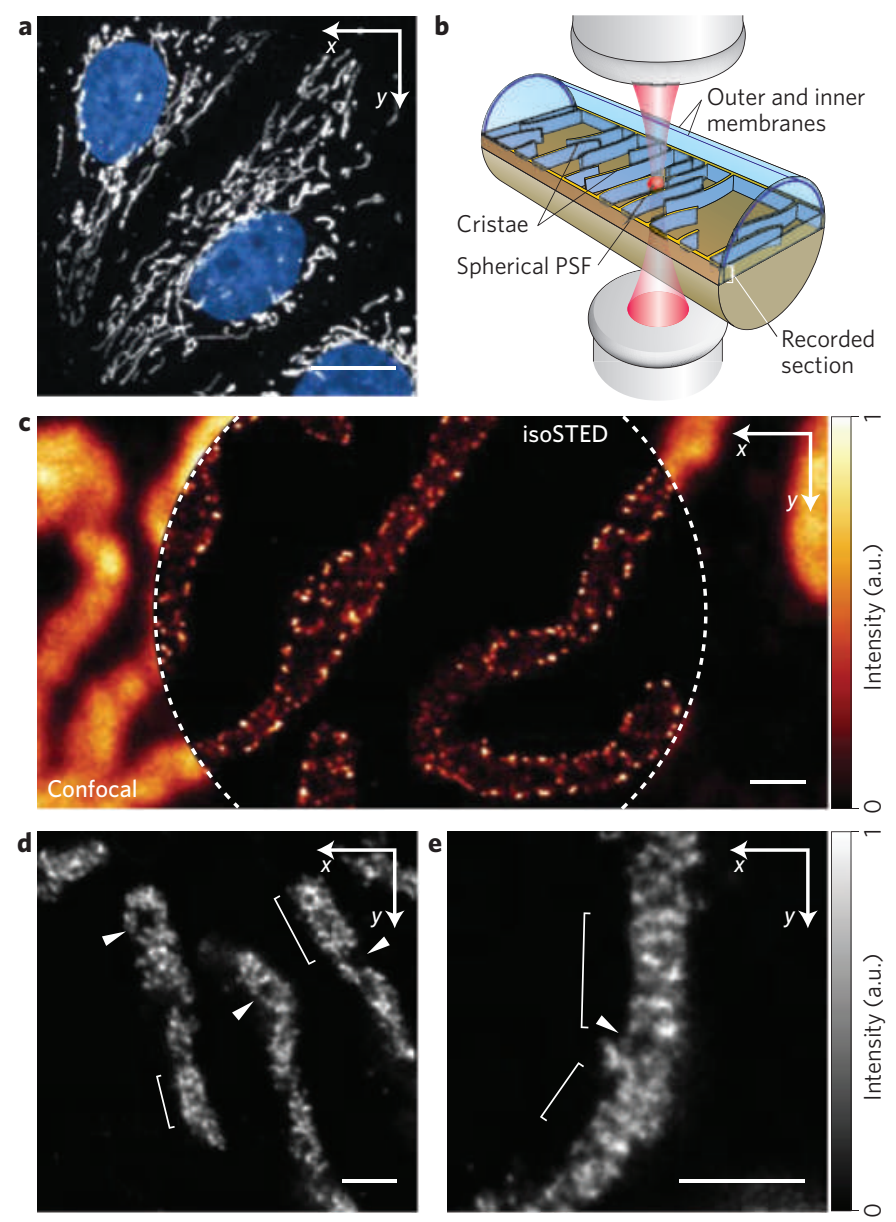

Figure 4 | Noninvasive 3D far-field fluorescence nanoscopy of the interior of mitochondria within integral cells. a, Confocal microscopy overview of a mammalian (PtK2) cell outlining the mitochondrial network (grey) and the nucleus (blue). b, Sketch of isoSTED nanoscope optically dissecting the interior of a mitochondrion with a nanospherical effective PSF. c, isoSTED nanoscopy resolves and correctly identifies Tom20 (translocase of the outer mitochondrial membrane) protein complexes at the outer mitochondrial membrane, whereas confocal microscopy fails to provide any positional information. The complexes represent the sites where cellular proteins enter the mitochondria. $\mathbf{d}, \mathbf{e}$, isoSTED images recorded at the mitochondrial equatorial plane reveal immunolabelled $F_{1} F_{0}$ ATPase proteins of the inner mitochondrial membrane and the cristae as essential structural elements of this organelle. The brackets indicate regions in which the cristae are perpendicularly oriented to the organelle axis, whereas the arrowheads point to inner mitochondrial regions devoid of cristae. Scale bars: $10 \mu \mathrm{m}$ (a) and $500 \mathrm{~nm}$ (c-e). Images d,e are reproduced with permission from ref. 49. (c) 2009 ACS.

photons, iPALM experiments showed the possibility of attaining a resolving power of $<22 \mathrm{~nm}$ and $<9 \mathrm{~nm}$ (FWHM) in the focal plane and along the optic axis, respectively. A clear advantage of improving the PALM/STORM 3D resolution with dual lenses over the earlier single-lens approaches, which rely on introducing asymmetries in the detection PSF, is that the lateral PSF width is not widened; the 4Pi detection PSF has virtually the same lateral cross-section as that of a single-lens epifluorescence microscope ${ }^{22}$. Hence, the localization in the focal plane is not compromised and the number of active (state A) molecules per area or volume need not be reduced.

The potential of iPALM to visualize biomolecules in three dimensions at nanometric resolution is exemplified in Fig. 5, which shows 

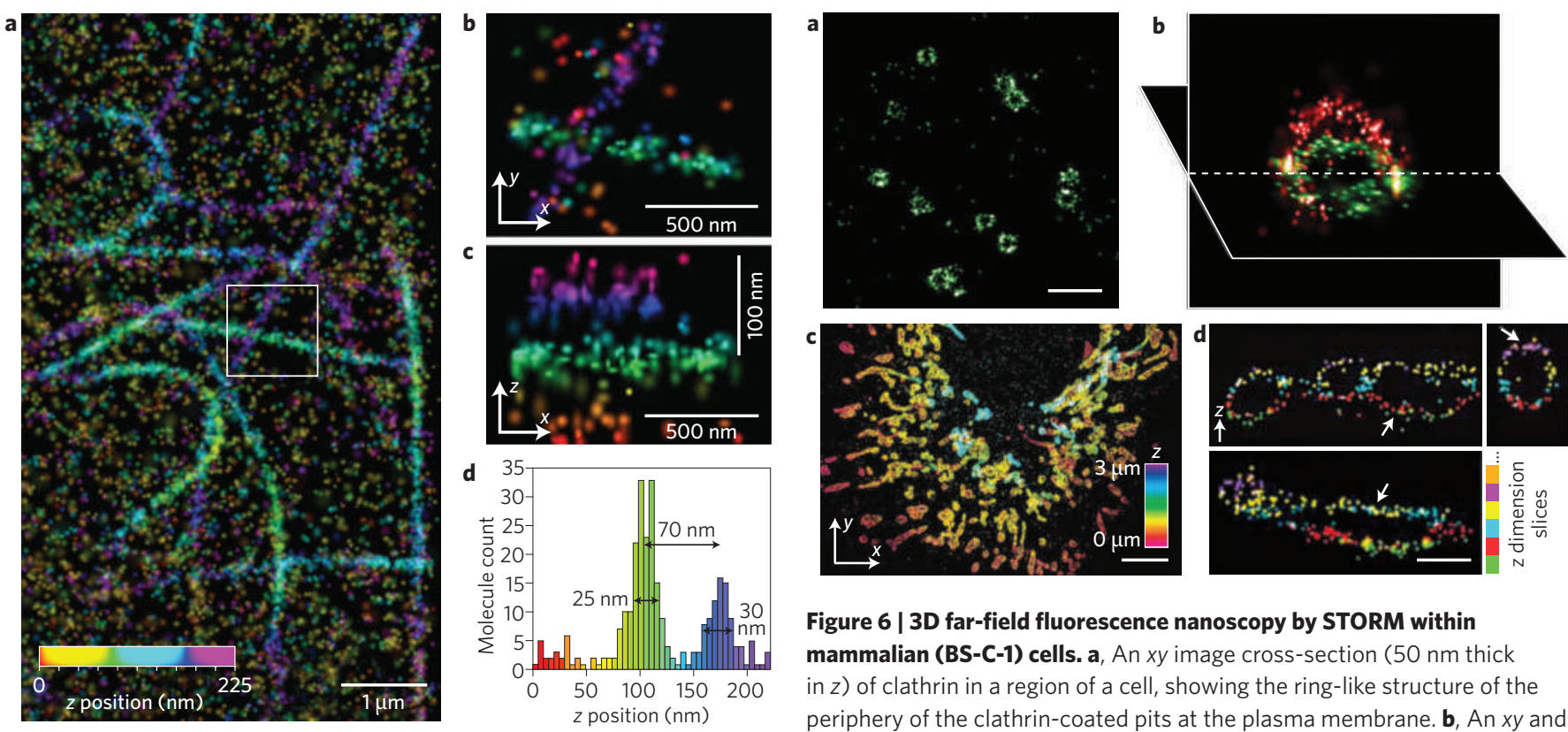

Figure 6 | 3D far-field fluorescence nanoscopy by STORM within mammalian (BS-C-1) cells. a, An $x y$ image cross-section ( $50 \mathrm{~nm}$ thick in $z$ ) of clathrin in a region of a cell, showing the ring-like structure of the periphery of the clathrin-coated pits at the plasma membrane. b. An $x y$ and $x z$ cross-section presented in 3D perspective, showing the half-spherical cage-like structure of a clathrin pit. c, 3D image of the mitochondrial

Figure 5 | 3D fluorescence nanoscopy with interferometric PALM. Microtubules in a mammalian (PtK1) cell expressing human $\alpha$-tubulin fused to a monomeric variant of the photoactivatable fluorescent protein KikGR. The visualization of the obtained axial resolution is enhanced by colour coding the image data depending on the position of the registered fluorophores in the $z$ direction. a, Large area overview. b, c, Magnified view of the area bound by the white box as $x y(\mathbf{b})$ and $x z$ (c) projections ( $z$ scale is magnified $\times 5$ ). $\mathbf{d}$, Histogram of $z$ positions of individually switched molecules in the boxed region. Each microtubule has a measured FWHM of $30 \mathrm{~nm}$, which is in accordance with the expectations from electron microscopy ( $25 \mathrm{~nm}$ plus label). Microtubules only $70 \mathrm{~nm}$ apart along the $z$ axis are separated by a pronounced dip, thus proving the resolving power provided by coherently detecting the fluorescence of individually switched fluorophores through opposing lenses. Reproduced with permission from ref. 44. (c) 2009 PNAS.

that microtubules at an axial distance of $\sim 70 \mathrm{~nm}$ are readily discerned $^{44}$. The method iPALM has also been able to resolve the dorsal and ventral plasma membranes and to visualize the arrangement of integrin receptors within the endoplasmic reticulum ${ }^{44}$, which have so far required electron microscopy. Although current implementations are limited to resolving structures thinner than $\sim \lambda / 2 n$, this restriction should be alleviated in the future. As a targeted modality in which the probing volume given by $\Delta \rho$ and $\Delta z$ can be directed to any part of the sample, isoSTED microscopy does not entail conceptual restrictions with regard to sample thickness. However, practical limitations of both targeted and stochastic approaches are posed by the free working distance of the lenses of $\sim 200 \mu \mathrm{m}$ and the (spherical) aberrations induced by the sample. Modern developments in active and adaptive optics should help advance both 3D nanoscopy methods using opposing lenses.

Comparison with other 3D nanoscopy schemes and outlook We note that increasing the 3D far-field optical nanoscopy resolution is possible with single-lens schemes as well as with dual lenses, both in STED microscopy ${ }^{11,50}$ and in PALM/STORM ${ }^{34,39,51,52}$. This stems from the fact that the resolution of both concepts is not limited by diffraction in any direction, but mainly determined by the $I / I_{s}$, or $m$ implemented. For example, in STORM a remarkable lateral and axial resolution combination of 20-30 $\mathrm{nm}$ and 50$60 \mathrm{~nm}$, respectively, has been demonstrated by encoding the $z$ axis

network, immuno-stained against the protein Tom20. The colour code indicates the position along the $z$ axis according to the colour scale bar. d, Vertical cross-sections of several regions in panel c, colour-coded by the $z$ coordinate of the $x y$ slices at which they were recorded. Arrows indicate horizontal segments of mitochondria appearing in adjacent slices along the $z$ axis; localizations from different slices are coloured differently. Scale bars: $500 \mathrm{~nm}$ (a), $5 \mu \mathrm{m}$ (c), $750 \mathrm{~nm}$ (d). Panels from refs. 51,52. Images a,b and $\mathbf{c}, \mathbf{d}$ are reproduced with permission from, respectively, ref. 51. (c) 2009 AAAS; and ref. 52. (c) 2009 NPG.

through astigmatism ${ }^{51,52}$. STORM 3D recordings (Fig. 6) of the clathrin-coated pits at the plasma membrane of a mammalian cell impressively revealed their spherical cage-like structure. Likewise, 3D STORM images of the mitochondrial network, immuno-stained against the protein Tom 20 in a mammalian cell, rendered the hollow structure of mitochondria. Defocus has also been effectively used for axial localization ${ }^{35}$. Similarly impressive resolution in the lateral and axial direction together with a larger depth of field has been shown by implementing a double-helix PSF ${ }^{39}$.

Clearly, these advances in single-lens 3D diffraction-unlimited nanoscopy remain highly important, because adding a second lens also adds complexity to the system. Furthermore, using opposing lenses will be possible only for transparent objects that are typically thinner than $200 \mu \mathrm{m}$ and accessible from two sides, such that the refractive index of the immersion system is adequately matched to that of the sample. Nonetheless, when applying the same $I / I_{s}$ or detecting the same $m$, having two opposing lenses always improves the $3 \mathrm{D}$ resolution further and concomitantly renders an isotropic nanoscale resolution at the highest possible level. It should be noted that irrespective of the brightness of the fluorescence marker or the specific set-up used, in single-molecule switching schemes such as PALM and STORM, combining the aperture of two opposing lenses for detection is expected to bring about a resolution gain of 1.4-fold in the focal plane and sixfold in the axial direction ${ }^{43}$.

The exploitation of opposing lenses will also benefit from the ample experience gained in the last two decades with $4 \mathrm{Pi}$ microscopy and $I^{5} \mathrm{M}$ or $\mathrm{I}^{5} \mathrm{~S}$. The application of these methods has shown that the benefits brought about by using two opposing lenses outweigh the additional complexity in many $\operatorname{cases}^{24-26,28}$, a notion that is corroborated by the progress reported herein. In fact, compact 
beam-scanning 4Pi microscopes with small modifications allow coherent dual-lens fluorescence excitation and collection, both in a point-scanning and in a widefield fashion, and have been commercially available since 2004 (ref. 29). Finally we note that, as isoSTED and iPALM are just representatives of two complementary nanoscopy modalities relying on fluorescence switching, one can expect other molecular switching mechanisms to benefit in much the same way from the coherent use of opposing lenses. For all these reasons, we expect dual-lens schemes to become central to 3D far-field optical imaging at the nanoscale in the near future.

\section{References}

1. Born, M. \& Wolf, E. Principles of Optics 7th edn (Cambridge University Press, 2002)

2. Alberts, B. et al. Molecular Biology of the Cell 4th edn (Garland Science, 2002).

3. Pohl, D. W., Denk, W. \& Lanz, M. Optical stethoscopy: Image recording with resolution $\lambda / 20$. Appl. Phys. Lett. 44, 651-653 (1984).

4. Lewis, A., Isaacson, M., Harootunian, A. \& Murray, A. Development of a 500 A resolution light microscope. Ultramicroscopy 13, 227-231 (1984).

5. Novotny, L. \& Hecht, B. Principles of nano-optics (Cambridge University Press, 2006).

6. Inouye, Y., Verma, P. \& Kawata, S. Plasmonics for near-field nano-imaging and superlensing. Nature Photon. 3, 388-394 (2009).

7. Pendry, J. B. Negative refraction makes a perfect lens. Phys. Rev. Lett. 85, 3966-3969 (2000)

8. Hell, S. W. \& Wichmann, J. Breaking the diffraction resolution limit by stimulated emission: stimulated emission depletion fluorescence microscopy. Opt. Lett. 19, 780-782 (1994).

9. Hell, S. W. \& Kroug, M. Ground-state depletion fluorescence microscopy, a concept for breaking the diffraction resolution limit. Appl. Phys. B 60, 495-497 (1995).

10. Hell, S. W. in Topics in Fluorescence Spectroscopy Vol. 5 (ed. J. R. Lakowicz) 361-422 (Plenum Press, 1997).

11. Klar, T. A., Jakobs, S., Dyba, M., Egner, A. \& Hell, S. W. Fluorescence microscopy with diffraction resolution limit broken by stimulated emission. Proc. Natl Acad. Sci. USA 97, 8206-8210 (2000).

12. Dyba, M. \& Hell, S. W. Focal spots of size $\lambda / 23$ open up far-field fluorescence microscopy at $33 \mathrm{~nm}$ axial resolution. Phys. Rev. Lett. 88, 163901 (2002).

13. Hell, S. W. Toward fluorescence nanoscopy. Nature Biotechnol. 21, 1347-1355 (2003).

14. Heintzmann, R., Jovin, T. M. \& Cremer, C. Saturated patterned excitation microscopy - A concept for optical resolution improvement. J. Opt. Soc. Am. A 19, 1599-1609 (2002)

15. Gustafsson, M. G. L. Nonlinear structured-illumination microscopy: Wide-field fluorescence imaging with theoretically unlimited resolution. Proc. Natl Acad. Sci. USA 102, 13081-13086 (2005).

16. Willig, K. I., Rizzoli, S. O., Westphal, V., Jahn, R. \& Hell, S. W. STEDmicroscopy reveals that synaptotagmin remains clustered after synaptic vesicle exocytosis. Nature 440, 935-939 (2006).

17. Betzig, E. et al. Imaging intracellular fluorescent proteins at nanometer resolution. Science 313, 1642-1645 (2006)

18. Rust, M. J., Bates, M. \& Zhuang, X. Sub-diffraction-limit imaging by stochastic optical reconstruction microscopy (STORM). Nature Meth 3, 793-796 (2006).

19. Hess, S. T., Girirajan, T. P. K. \& Mason, M. D. Ultra-high resolution imaging by fluorescence photoactivation localization microscopy. Biophys. J. 91, 4258-4272 (2006).

20. Egner, A. et al. Fluorescence nanoscopy in whole cells by asnychronous localization of photoswitching emitters. Biophys. J. 93, 3285-3290 (2007).

21. Hell, S. W. Double-scanning confocal microscope. European patent 0491289 (1990/1992).

22. Hell, S. \& Stelzer, E. H. K. Properties of a 4Pi-confocal fluorescence microscope. J. Opt. Soc. Am. A 9, 2159-2166 (1992).

23. Gustafsson, M. G. L., Agard, D. A. \& Sedat, J. W. Sevenfold improvement of axial resolution in 3D widefield microscopy using two objective lenses. Proc. SPIE 2412, 147-156 (1995).

24. Hell, S. W., Schrader, M. \& van der Voort, H. T. M. Far-field fluorescence microscopy with three-dimensional resolution in the $100 \mathrm{~nm}$ range. J. Microsc. 185, 1-5 (1997).

25. Gustafsson, M. G. L., Agard, D. A. \& Sedat, J. W. I5: 3D widefield light microscopy with better than $100 \mathrm{~nm}$ axial resolution. J. Microsc. 195, 10-16 (1999).

26. Shao, L. et al. I5S: Wide-field light microscopy with $100 \mathrm{~nm}$ scale resolution in three dimensions. Biophys. J. 94, 4971-4983 (2008).

27. Hell, S. W. Far-field optical nanoscopy. Science 316, 1153-1158 (2007).
28. Gugel, H. et al. Cooperative 4Pi excitation and detection yields 7 -fold sharper optical sections in live cell microscopy. Biophys. J. 87, 4146-4152 (2004).

29. Bewersdorf, J., Bennett, B. T. \& Knight, K. L. H2AX chromatin structures and their response to DNA damage revealed by $4 \mathrm{Pi}$ microscopy. Proc. Natl Acad. Sci. USA 103, 18137-18142 (2006).

30. Gustafsson, M. G. L. \& Clarke, J. Scanning force microscope springs optimized for optical-beam deflection and with tips made by controlled fracture. J. Appl. Phys. 76, 172-181 (1994).

31. Hofmann, M., Eggeling, C., Jakobs, S. \& Hell, S. W. Breaking the diffraction barrier in fluorescence microscopy at low light intensities by using reversibly photoswitchable proteins. Proc. Natl Acad. Sci. USA 102, 17565-17569 (2005).

32. Sharonov, A. \& Hochstrasser, R. M. Wide-field subdiffraction imaging by accumulated binding of diffusing probes. Proc. Natl Acad. Sci. USA 103, 18911-18916 (2006).

33. Shroff, H. et al. Dual-color superresolution imaging of genetically expressed probes within individual adhesion complexes. Proc. Natl Acad. Sci. USA 104, 20308-20313 (2007).

34. Juette, M. F. et al. Three-dimensional sub-100 nm resolution fluorescence microscopy of thick samples. Nature Meth. 5, 527-529 (2008).

35. Bates, M., Huang, B., Dempsey, G. P. \& Zhuang, X. Multicolor superresolution imaging with photo-switchable fluorescent probes. Science 317, 1749-1753 (2007).

36. Zhuang, X. Nano-imaging with STORM. Nature Photon. 3, 365-367 (2009).

37. Rittweger, E., Wildanger, D. \& Hell, S. W. Far-field fluorescence nanoscopy of diamond color centers by ground state depletion. Europhys. Lett. 86, 14001-14006 (2009)

38. Biteen, J. S. et al. Super-resolution imaging in live caulobacter crescentus cells using photoswitchable EYFP. Nature Meth. 5, 947-949 (2008).

39. Pavani, S. R. P. et al. Three-dimensional, single-molecule fluorescence imaging beyond the diffraction limit by using a double-helix point spread function. Proc. Natl Acad. Sci. USA 106, 2995-2999 (2009).

40. van de Linde, S., Kasper, R., Heilemann, M. \& Sauer, M. Photoswitching microscopy with standard fluorophores. Appl. Phys. B 93, 725-731 (2008)

41. Heilemann, M. et al. Subdiffraction-resolution fluorescence imaging with conventional fluorescent probes. Angew. Chem. Int. Ed. 47, 6172-6176 (2008)

42. Dyba, M., Jakobs, S. \& Hell, S. W. Immunofluorescence stimulated emission depletion microscopy. Nature Biotechnol. 21, 1303-1304 (2003).

43. Middendorff, C.v., Egner, A., Geisler, C., Hell, S. W. \& Schönle, A. Isotropic 3D nanoscopy based on single emitter switching. Opt. Express 16, 20774-20788 (2008).

44. Shtengel, G. et al. Interferometric fluorescent super-resolution microscopy resolves 3D cellular ultrastructure. Proc. Natl Acad. Sci. USA 106, 3125-3130 (2009).

45. Schmidt, R. et al. Spherical nanosized focal spot unravels the interior of cells. Nature Meth. 5, 539-544 (2008).

46. Lemmer, P. et al. SPDM - Light microscopy with single molecule resolution at the nanoscale. Appl. Phys. B 93, 1-12 (2008).

47. Egner, A., Schrader, M. \& Hell, S. W. Refractive index mismatch induced intensity and phase variations in fluorescence confocal, multiphoton and $4 \mathrm{Pi}-$ microscopy. Opt. Commun. 153, 211-217 (1998).

48. Ullal, C. K., Schmidt, R., Hell, S. W. \& Egner, A. Block copolymer nanostructure mapped by far-field optics. Nano Lett. 9, 2497-2500 (2009).

49. Schmidt, R. et al. Mitochondrial cristae revealed with focused light. Nano Lett. 9, 2508-2510 (2009).

50. Harke, B., Ullal, C. K., Keller, J. \& Hell, S. W. Three-dimensional nanoscopy of colloidal crystals. Nano Lett. 8, 1309-1313 (2008).

51. Huang, B., Wang, W., Bates, M. \& Zhuang, X. Three-dimensional superresolution imaging by stochastic optical reconstruction microscopy. Science 319, 810-813 (2008).

52. Huang, B., Jones, S. A., Brandenburg, B. \& Zhuang, X. Whole-cell 3D STORM reveals interactions between cellular structures with nanometer-scale resolution. Nature Meth. 5, 1047-1052 (2008).

\section{Acknowledgements}

Substantial contributions to the isoSTED imaging applications reviewed in this paper are from Chaitanya Ullal (block co-polymers), as well as Christian Wurm and Stefan Jakobs (mitochondria). We also thank Andreas Schönle, Claas v. Middendorf and Jan Keller for helpful discussions and Jaydev Jethwa for critical reading. This work was supported by grants of the Deutsche Forschungsgemeinschaft to A.E. and S.W.H (SFB 755).

\section{Additional information}

Details accompany the full-text HTML version of the paper at www.nature.com/ naturephotonics. Reprints and permission information is available online at http://npg. nature.com/reprintsandpermissions/. Correspondence and requests for materials should be addressed to S.W.H. 